\title{
RFID-BASED WAREHOUSE MANAGEMENT SYSTEM PROTOTYPING USING A HETEROGENEOUS TEAM OF ROBOTS
}

\author{
ARTUR KHAZETDINOV, ANDREY ALEKSANDROV, AUFAR ZAKIEV and EVGENI MAGID \\ Laboratory of Intelligent Robotic Systems, Intelligent Robotics Department, \\ Higher Institute of Information Technology and Intelligent Systems, Kazan Federal University, 35 \\ Kremlevskya street, Kazan, 420008, Russia \\ E-mail: ark@it.kfu.ru, andre16reg@gmail.com,zaufar@it.kfu.ru,magid@it.kfu.ru \\ kpfu.ru/robolab.html \\ KUO-HSIEN HSIA \\ Department of Electrical Engineering, National Yunlin University of Science and Technology, \\ Tainan City, 74448, Taiwan \\ E-mail: khhsia@yuntech.edu.tw
}

\begin{abstract}
Robotic warehouse management is a promising field of research with a high practical applicability. Typically, warehouses are strictly organized and controlled environments with a high potential for rapid robot deployments. To provide system robustness every robotic deployment should be tested in advance using simulations. Such virtual testing environment should correspond well with a real world warehouse environment and be consistent with international and national standards. In this paper, we present a warehouse Gazebo simulation that was constructed in accordance with Government Standards of Russian Federation and international standards. We implemented a simplified Radio Frequency IDentification (RFID) transmitter-receiver mechanism within the Gazebo simulator and tested it in a virtual warehouse environment using a heterogeneous team of TIAGo Base mobile robot and PX4-based UAV. Our constructed virtual warehouse environment and RFID mechanism models are open-source for academic community use.
\end{abstract}

\section{Introduction}

Modern warehouse automation technologies promise to completely change methods of warehouse management. Automatic goods storage and order collection increase warehouse efficiency and safety. Robotics is essential for the vast majority of warehouse automation approaches: robots could be used for cargo transfer, goods inventory, safety management, and other tasks. In the observable future, robotics will continue increasing its role in order to resolve typical issues in warehouse management. ${ }^{1}$ One of warehouse management tasks, which has high efficiency of robotic deployment, is goods inventory. An enterprise warehouse inventory requires a significant amount of resources since usually such warehouses occupy large areas, which are populated with racks of 10 or more meters height and store thousands of goods. ${ }^{2}$ In addition to supreme time consumption, an inventory task has a high risk of injury for human staff, e.g., due to a necessity of climbing folding stairs in order to reach a majority of racks' shelves. In such conditions, autonomous robots allow performing inventory faster, more often and more accurately than human workers could while simultaneously increasing warehouse staff safety.

To guarantee a robotic system robustness the system should be thoroughly tested prior to its deployment. The first stages of development and testing could be performed in simulations as they provide a resource-saving way of new hardware and software evaluation. While there is a broad variety of 3D simulators for robotic research, recently the Gazebo ${ }^{3}$ became one of the most popular simulators due to its seamless integration with the Robot Operating System (ROS) framework. It has strong integration with multiple modern robots, their $3 \mathrm{D}$ models and built-in navigation features. ${ }^{4-6}$ The Gazebo has a number of built-in de- 
fault environments and basic mechanisms to construct new environments. ${ }^{7}$ Moreover, some user-created open-source packages significantly increase the power of new environment construction, which allows to automate the process and integrate various constraints into the environment. ${ }^{3}$ Even though the last versions of Gazebo include various sensors that could be directly used within virtual environments, it does not provide a Radio Frequency IDentification (RFID) sensor (reader or receiver) and RFID tags (transmitters) built-in software.

This paper presents a virtual warehouse Gazebo simulation and RFID transmitterreceiver mechanism implementation in Gazebo. The virtual warehouse was constructed in accordance with Government Standards of the Russian Federation and international standards. ${ }^{8,9}$ A simplified RFID mechanism implementation includes virtual RFID sensors and virtual RFID tags that communicate using our built-in software. The mechanism was tested in a virtual warehouse environment using a heterogeneous team of a TIAGo Base ${ }^{10}$ unmanned ground vehicle (UGV) and a PX4-based unmanned aerial vehicle (UAV).

The rest of the paper is organized as follows. Section 2 is dedicated to related work on robotic warehouse management. Section 3 describes warehouse and RFID device requirements. Section 4 presents virtual warehouse and virtual RFID mechanism implementation. Section 5 demonstrates virtual experiments. Conclusions appear in the last section.

\section{Related Work}

Various visual and non-visual markers (tags) are used to automate inventory processes. These tags label warehouse infrastructure, including mobile carts, racks, individual shelves, items, and goods. Commonly, RFID tags ${ }^{11}$, barcodes, QR-codes ${ }^{12}$, or fiducial markers ${ }^{13}$ could be used. $\mathrm{In}^{11,14}$ authors discussed a system for inventory tasks automation based on RFID technology. A UAV was equipped with an RFID reader to read RFID tags that were attached to warehouse goods. Such labeling improves an inventory process in terms of time and precision and does not pose limitations on visibility conditions within a warehouse for tags' recognition. An improved version of RFID, called RFly, was developed and successfully deployed on Parrot Bebop UAV ${ }^{15}$. However, the Gazebo simulator has no built-in RFID reader and tag implementation, which is not trivial due to its non-visual approach.

$\operatorname{In}^{16}$ the authors discussed high-level design considerations and implementation challenges of a multi-UAV ${ }^{17}$ system equipped with RFID readers, which should aid in warehouse inventory automation. However, robust indoor UAV navigation is still a major issue ${ }^{18}$. Therefore, researchers tend to use manual flight control and to split a flight controller and an RFID reader in order to simplify their software ${ }^{19}$.

Another approach to an indoor navigation for an autonomous warehouse inventory applies a mobile UGV as a moving platform with a robust sensor perception and navigation. ${ }^{1,20}$ It serves as a carrying platform and a ground reference for an indoor flight of a UAV, which is used as a mobile scanner (reader) with six degrees of freedom. The UGV (with the UAV on its top) navigates among rows of racks. The UGV stops near each rack and the UAV takes off to fly vertically to scan goods in the rack. Since the UGV is used as a navigation reference point, the UAV adjusts its localization continuously without getting lost.

While most of the above-mentioned authors validated their approaches in artificial smallsize laboratory environments, these approaches do require in-depth testing in large-size environments before actual deployment into a real commercial warehouse environment. However, to the best of our knowledge, no comprehensive RFID-based warehouse simulation exists at this moment. Therefore, to perform a meaningful evaluation of warehouse inventory approaches, a valid virtual environment with a proper RFID mechanism interaction, robot locomotion and standardized proportions of a typical real-world setup is required. 


\section{Warehouse environment requirements}

\subsection{Virtual warehouse setup}

Virtual environments are fast to build and do not require much resources to create. In our project warehouse environments were constructed in accordance with Government

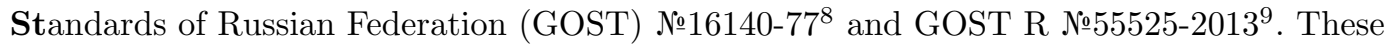
standards are obligatory for all warehouses in the Russian Federation. The following properties are defined for warehouses by GOSTs and international practices:

- The minimum distance between a warehouse wall and any rack is $0.7 \mathrm{~m}$. This is enough for a person to pass along any wall of the warehouse in accordance with GOST №16140-77.

- The width of a corridor between racks is between 2.5 and $3.7 \mathrm{~m}$. This width allows a forklift to turn at 90 degrees in accordance with GOST R №55525-2013. An example of a corridor is shown in Fig. 1.

- Warehouse goods are placed on standardized euro-pallets. Such goods' storage is widely accepted around the world.

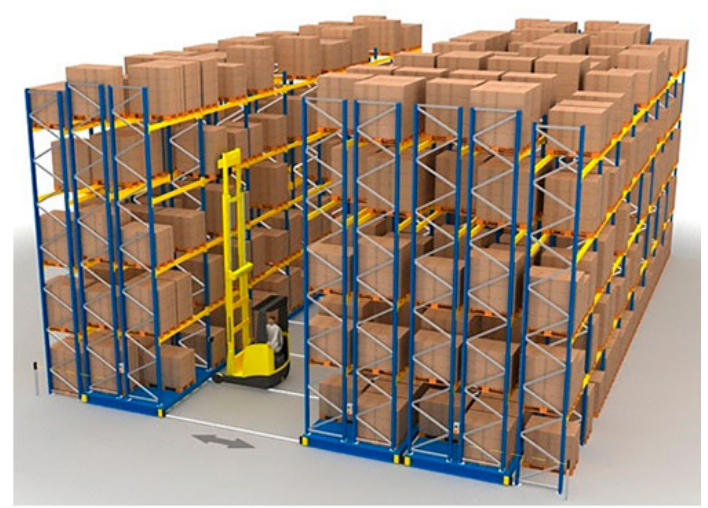

Fig. 1: Warehouse corridor example complied with GOST requirements. The image is borrowed from promstellage.ru.

\subsection{RFID system}

A Radio Frequency IDentification (RFID) is a method for automatic identification of objects. It distinguishes objects using radio transponders, commonly, RFID tags. Data stored in an RFID tag could be read by an RFID reader at a distance within a certain range. An RFID tag consists of a chip and an antenna. When an RFID tag is within an RFID reader range, it transmits data that are stored in the tag. Next, the RFID reader transmits the received data to a server. A standard RFID system includes a tag, a reader, an antenna, and a software.

An RFID reader is an essential element of an RFID system. It emits radio waves that activate RFID tags. Activated RFID tag emits radio waves with data, which are stored in the tag. An RFID reader detects tag response radio waves and processes the data, which allows to identify the detected RFID tag uniquely. RFID readers that are used in warehouses could be stationary or mobile. Stationary RFID readers are mounted on walls, doors, and other static objects and have a long range for RFID tags reading. Most often, they are used in large enterprises with high traffic of goods. Mobile RFID readers are used as portable devices that are powered from batteries and have a small weight (from 80 g.). They have built-in antennas and a connector for installing an additional antenna, while their reading 
range is much shorter relatively to a stationary RFID reader range. An additional RFID antenna increases a range of an RFID reader in a particular direction and thus they could be effectively used when a direction toward an RFID tag is known.

RFID tags are divided by a type of power supply into passive (without a power supply), active, and semi-passive (the latter two are both with their own power supply). A reading range depends on a power consumption level and a tag's operating frequency. Higher frequency and higher power consumption imply a longer tag reading range. Commonly, RFID tags operating frequencies are divided into a low frequency (LF, 125-134 kHz), a high frequency (HF, $13.56 \mathrm{MHz}$ ), and an ultra-high frequency (UHF, 860-960 MHz). Their approximate reading ranges are presented in Table 1.

Table 1: Correspondence between a frequency and a passive reading range. ${ }^{21}$

\begin{tabular}{lll}
\hline Type & Frequency & Reading range \\
\hline Low frequency (LF) & $125-134 \mathrm{kHz}$ & Up to $10 \mathrm{~cm}$ \\
High frequency (HF) & $1.75-13.56 \mathrm{MHz}$ & Up to $20 \mathrm{~cm}$ \\
Ultra high frequency (UHF) & $860-960 \mathrm{MHz}$ & Up to $300 \mathrm{~cm}$ \\
\hline
\end{tabular}

A UAV-attached RFID reader for a warehouse should display the following properties:

- A low weight. A typical UAV has a limited payload of up to about $1 \mathrm{~kg}$.

- A UHF operating frequency. This maximizes a reading range.

- An integrated own battery. This allows easy integration into a UAV system.

- ROS integration. ${ }^{22}$ This is desirable for seamless software integration into a UAV control system (MAVLink, ROS, etc.).

For example, ThingMagic USB Pro RFID Reader ${ }^{23}$ meets all of the above requirements: it weights $160 \mathrm{~g}$, has a built-in battery, supports a UHF frequency and external antennas. The external antennas increase reading range up to 10 meters. Self-adhesive UHF tags could be used as RFID tags since they have a rather low cost of approximately $\$ 0.86$ per unit. This RFID reader was used as a reference during a receiver modeling in the Gazebo simulator.

\section{Virtual Experiments}

\subsection{Warehouse modelling}

We constructed a virtual warehouse environment that fulfills all the requirements of modern standards, which were presented in Section 3.1. It is designed to be reusable, therefore, all created 3D models are modular, universal and their locations within the virtual warehouse are easy to adjust.

\subsection{RFID system simulation}

The RFID system is implemented in the Gazebo simulator using a wireless receiver and wireless transmitter primitives. A wireless receiver is used as an RFID reader, and a wireless transmitter serves as an RFID tag. When the transmitter is within the receiver range, the receiver gets data that are stored in the transmitter. Basic data that are stored by the transmitter are a unique identifier (Name or ID), a transmitter operating frequency and a signal level. The signal level is calculated based on Okumura-Hata model. ${ }^{24}$ To preserve the model simplicity, we modeled the RFID reader appearance as a red cube of the corresponding to the ThingMagic USB Pro RFID Reader weight and attached it to a UAV bottom (see 

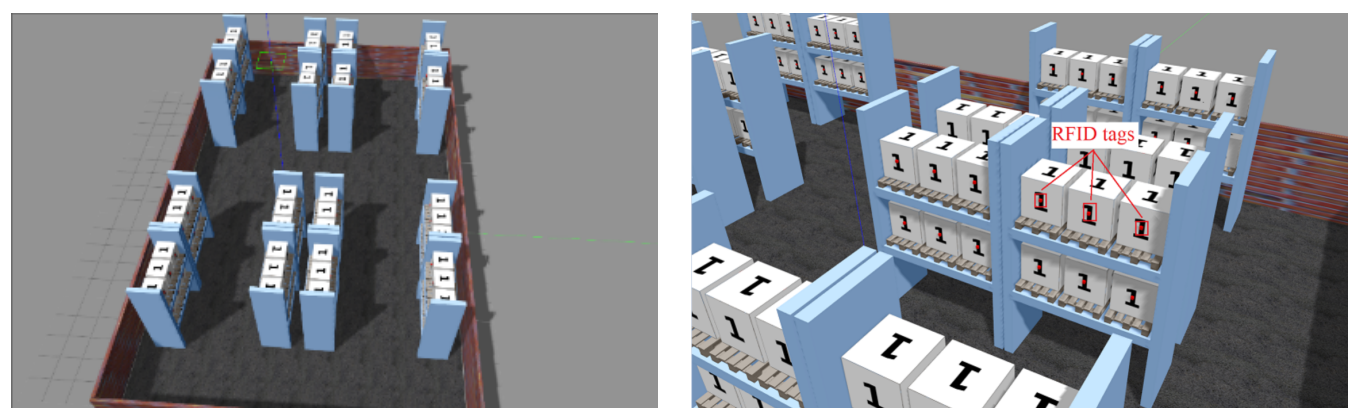

Fig. 2: A block of a warehouse model in the Gazebo simulator (left) with goods placed on racks. Each good has a unique RFID tag (right).

Fig. 4). The receiver continuously monitors environment for transmitters. If the transmitter and the receiver frequency correspond and they are within each others' range, the receiver obtains data stored by the transmitter. RFID tag visual representation is shown in Fig. 2.

\subsection{Mobile robots}

TIAGo Base is a mobile robot developed by PAL-Robotics. It is designed to have simultaneously a minimum footprint and a maximum payload. It is equipped with a laser range finder (LRF), inertial measurement unit (IMU) and ultrasonic sensors. The robot is integrated into the ROS framework and uses the ROS navigation stack for localization, path planning, and simultaneous localization and mapping (SLAM) ${ }^{25}$ The manufacturer also provides ROS packages for fully operational simulations with the TIAGo Base robot. Packages include CAD models (the robot model is shown in Fig. 3) and identical ROS packages for extensive testing in virtual environments. ${ }^{26}$
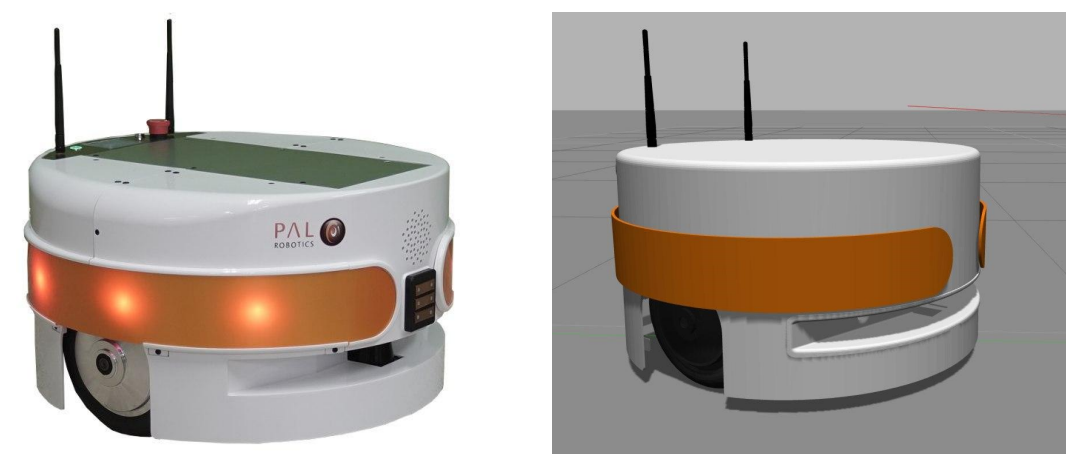

Fig. 3: The TIAGo Base mobile robot (left) and its model in the Gazebo simulator (right).

We used a UAV that is based on PX4 and PIXHAWK technologies. PX4 $4^{27}$ is opensource hardware for unmanned aerial vehicles control. PX4 software provides a flexible and unified set of tools for UAV developers and such unification significantly speeds up software development and decreases maintenance costs. PIXHAWK is an open hardware project used for constructing UAVs for academic and developer purposes. ${ }^{28}$ It seamlessly supports PX4 software and has high extensibility. The PX4-based UAV of Laboratory of Intelligent Robotic Systems (LIRS) at Kazan Federal University, its developed 3D-model and its joint formation with TIAGo Base are shown in Fig. 4. 

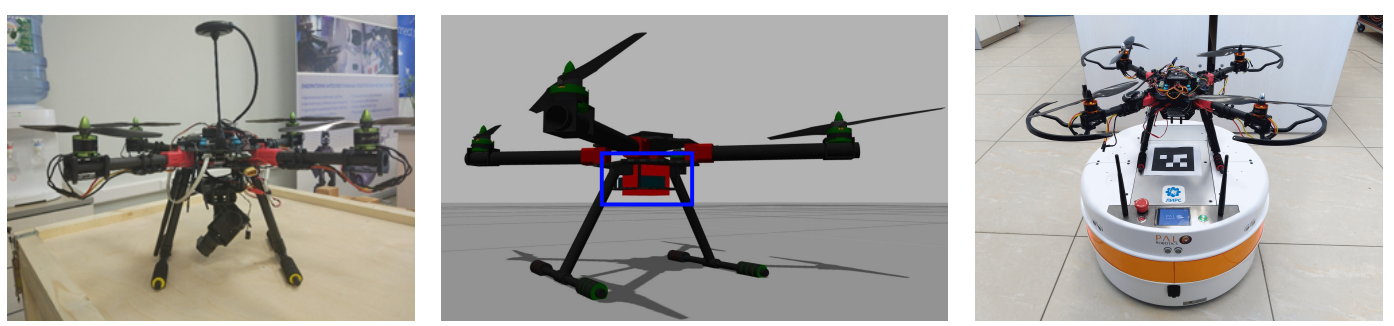

Fig. 4: The PX4-LIRS UAV (left) and its model in the Gazebo simulator with the RFID reader (middle). The RFID reader is highlighted with the blue rectangle. The TIAGo Base UGV with the ArUco tag 16h3 ID-198 on its top and the PX4-LIRS UAV (right).

\subsection{Virtual experiments setup}

All experiments were conducted in the virtual warehouse that was described in Section 4.1, with TIAGo Base UGV and PX4-LIRS UAV. Before the experiments, we constructed a map of the virtual environment using TIAGo Base robot with gmapping ROS package mapping algorithm. ${ }^{29}$ The resulting map is shown in Fig. 5a. The robot localizes within the map during experiments using AMCL ${ }^{30}$ method, which is encapsulated in amcl ROS package. It initially assumes a uniform probability of the robot location within the map as it is shown in Fig. 5b. This probability gradually converges to a particular position as the robot moves through the environment and localizes itself (the resulting distribution is shown in Fig. 5c).

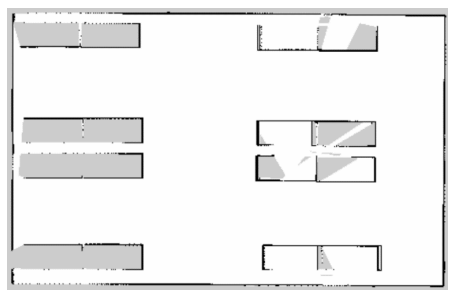

(a) The warehouse model map constructed by the TIAGo Base UGV with gmapping algorithm. lator.

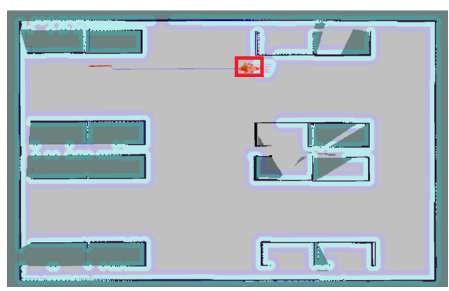

(c) The AMCL filter particles distribution during the robot movement.

Fig. 5: The constructed map and UGV localization process during navigation in the developed environment.

The TIAGo Base serves as a carrier that moves the PX4-LIRS UAV between racks inside the warehouse. The UAV has a mounted PX4FLOW ${ }^{31}$ optical flow camera with $752 \times 480$ pixels resolution, which faces downwards. Using this camera the UAV tracks the TIAGo Base in order to precisely land on the UGV's top. The tracking is performed using an ArUco marker, ${ }^{32}$ which is placed on the top flat surface of the TIAGo Base (shown in Fig. 4, on the right). Among thousands of available marker options, ArUco marker of $16 \mathrm{~h} 3$ type was selected due to its validated resistance to rotations. ${ }^{13}$

\subsection{Virtual experiments workflow}

At the start of virtual experiments, the TIAGo Base UGV spawns in a warehouse's corner. The PX4-LIRS UAV is initially placed on a planar top surface of the UGV. As input data, the UGV receives a set of goal points, which are located next to each warehouse rack. Each goal point defines a position where the UGV should deliver the UAV in order to further perform inventory of a particular rack. The UGV uses a predefined map of the warehouse 
(an example is shown in Fig. 5a) and global_planner ROS package to determine a path to a current goal point. After reaching the goal point, a ground station activates the UAV node. The UAV flies vertically upwards (Fig. 6, on the right). During the flight, it scans RFID tags and sends received data (tag ID and its height) to the UGV. ${ }^{33}$ These data are stored in the TIAGo Base's memory. The collected data is enough to determine each good's location in the warehouse. After the UAV reaches a height of $2 \mathrm{~m}$ (which is set as a maximum rack height of the model), it stays in the air for 20 seconds and then lands back on the UGV top. After that, the UGV moves to the next point until all goal points are covered. Upon accomplishing the task (all goals) the UGV returns to the starting point. The autonomous inventory operation architecture is schematically depicted in Fig. 6, on the left. Since each item of the warehouse has a unique RFID ID, multiple scans of the same RFID tag do not decrease automatic inventory performance but on the contrary, increases its precision.
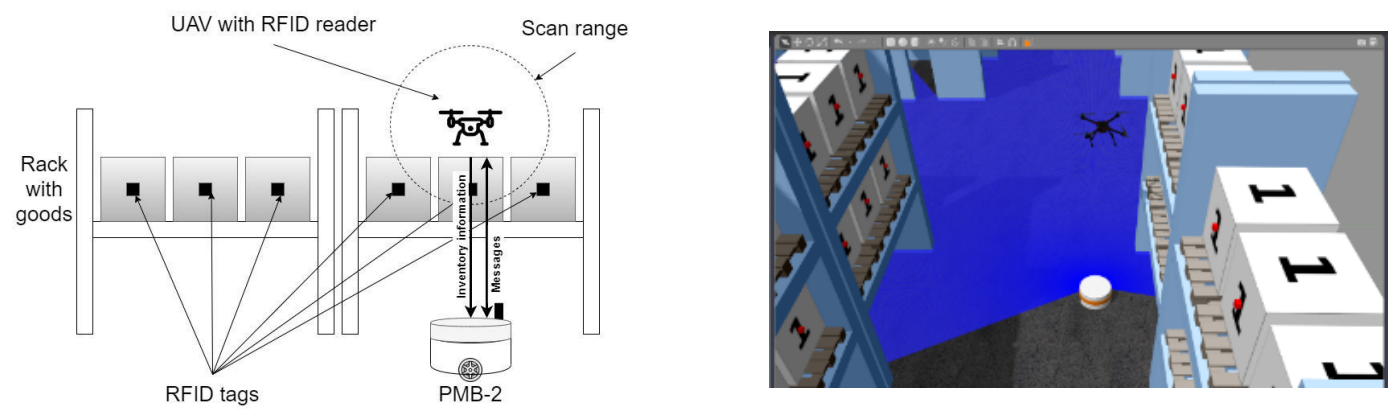

Fig. 6: Autonomous warehouse inventory operation architecture (left) and the UAV takeoff and scanning of RFID tags at a goal point of the UGV (right).

\section{Conclusions}

This paper presented a warehouse Gazebo simulation that was constructed in accordance with Government Standards of the Russian Federation and international standards. A simplified Radio Frequency IDentification (RFID) transmitter-receiver mechanism was implemented in the Gazebo simulator. Its performance was successfully evaluated in a virtual warehouse environment using a heterogeneous team of a TIAGo Base UGV and a PX4based UAV. Cooperative robots' activities during the virtual experiments included UGV autonomous mapping, localization and navigation, and UAV autonomous flight that utilized the developed RFID receiver (UAV-attached sensor) and multiple transmitters (RFID tags). The contribution of this paper is open-source and is available for a download from the LIRS repository on the GitLab platform, for free use by the academic community: https://gitlab.com/LIRS_Projects/Pixhawk-quadrotor-control.

\section{Acknowledgments}

This work was supported by the Russian Foundation for Basic Research (RFBR), project ID 19-58-70002.

\section{References}

1. F. Guérin, F. Guinand, J.-F. Brethé and H. Pelvillain, Towards an autonomous warehouse inventory scheme, in Symposium Series on Computational Intelligence, (IEEE, 2016).

2. A. Saveliev, M. Uzdiaev and M. Dmitrii, Aggressive action recognition using 3d cnn architectures, in International Conference on Developments in eSystems Engineering (DeSE), 2019. 
3. B. Abbyasov, R. Lavrenov, A. Zakiev, K. Yakovlev, M. Svinin and E. Magid, Automatic tool for gazebo world construction: from a grayscale image to a $3 \mathrm{~d}$ solid model, in International Conference on Robotics and Automation (ICRA), pp. 7226-7232, 2020.

4. Y. Bai, K. Asami and M. Svinin, Toward cooperative multi-robot control for detecting and tracking an expanding flood area, in International Conference on Developments in eSystems Engineering (DeSE), 2019.

5. I. Moskvin and R. Lavrenov, Modeling tracks and controller for servosila engineer robot, in Smart Innovation, Systems and Technologies, 2020.

6. F. G. Rodriguez-Telles, L. A. T. Mendez and E. A. M. Garcia, A fast floor segmentation algorithm for visual-based robot navigation, in Int. Conf. on Computer \& Robot Vision, 2013.

7. R. Lavrenov, A. Zakiev and E. Magid, Automatic mapping and filtering tool: From a sensorbased occupancy grid to a 3d gazebo octomap, in International Conference on Mechanical, System and Control Engineering (ICMSC), pp. 190-195, 2017.

8. GOST 16140-77 Collapsible racks http://docs.cntd.ru/document/gost-16140-77.

9. GOST R 55525-2013 Warehouse equipment http://docs.cntd.ru/document/1200103726.

10. Mobile robot PMB-2 http://wiki.ros.org/Robots/PMB-2.

11. K. Finkenzeller, RFID handbook: fundamentals and applications in contactless smart cards, radio frequency identification and near-field communication (John wiley \& sons, 2010).

12. H. Cho, D. Kim, J. Park, K. Roh and W. Hwang, 2D barcode detection using images for droneassisted inventory management, in International Conference on Ubiquitous Robots, 2018.

13. A. Zakiev, K. Shabalina, T. Tsoy and E. Magid, Pilot virtual experiments on aruco and artag systems comparison for fiducial marker rotation resistance, in Smart Innovation, Systems and Technologies, vol. 154, pp. 455-464, 2020.

14. J. Ong, Mobile rfid system for inventory automation, PhD Thesis, MIT2008.

15. Y. Ma, N. Selby and F. Adib, Drone relays for battery-free networks, in Proceedings of the Conference of the ACM Special Interest Group on Data Communication, 2017.

16. J. H. Ong, A. Sanchez and J. Williams, Multi-UAV system for inventory automation, in 2007 1st Annual RFID Eurasia, (IEEE, 2007).

17. A. Zakiev, T. Tsoy and E. Magid, Swarm robotics: Remarks on terminology and classification, in International Conference on Interactive Collaborative Robotics, pp. 291-300, 2018.

18. K. Yakovlev, V. Khithov, M. Loginov and A. Petrov, Distributed control and navigation system for quadrotor uavs in GPS-denied environments, in Intelligent Systems' 2014, (Springer, 2015).

19. S. M. Bae, K. H. Han, C. N. Cha and H. Y. Lee, Development of inventory checking system based on UAV and RFID in open storage yard, in International Conference on Information Science and Security, (IEEE, 2016).

20. Y. Lyu, Hybrid Aerial and Ground-based Mobile Robot for Retail Inventory 2018.

21. S. A. Weis, System 2, 1 (2007).

22. M. Quigley, K. Conley, B. Gerkey, J. Faust, T. Foote, J. Leibs, R. Wheeler and A. Y. Ng, ROS: an open-source Robot Operating System, in ICRA workshop on open source software, 2009.

23. Thingmagic_usbpro http://wiki.ros.org/thingmagic_usbpro.

24. A. Medeisis and A. Kajackas, On the use of the universal Okumura-Hata propagation prediction model in rural areas, in 51st Vehicular Technology Conference Proceedings, (IEEE, 2000).

25. N. Simakov, R. Lavrenov, A. Zakiev, R. Safin and E. A. Martínez-García, Modeling usar maps for the collection of information on the state of the environment, in International Conference on Developments in eSystems Engineering (DeSE), pp. 918-923, 2019.

26. N. Koenig and A. Howard, Design and use paradigms for gazebo, an open-source multi-robot simulator, in International Conference on Intelligent Robots and Systems, (IEEE, 2004).

27. PX4 Development Guide https://dev.px4.io/en/.

28. A. Yatsun, B. Lushnikov and O. Emelyanova, Motion control automation in the quadcopter convertiplane in a transient mode, in International Russian Automation Conference, 2018.

29. P. Wang, Z. Chen, Q. Zhang and J. Sun, IFAC-PapersOnLine 49, 168 (2016), Elsevier.

30. D. Fox, W. Burgard, F. Dellaert and S. Thrun, AAAI/IAAI 1999, 2 (1999).

31. PX4FLOW https://docs.px4.io/en/sensor/px4flow.html.

32. M. F. Sani, Automatic navigation and landing of an indoor AR. drone quadrotor using ArUco marker and inertial sensors, in Int. Conf. on Computer and Drone Applications, 2017.

33. A. Pashkin, R. Lavrenov, A. Zakiev and M. Svinin, Pilot communication protocols for group of mobile robots in usar scenarios, in International Conference on Developments in eSystems Engineering (DeSE), pp. 37-41, 2019. 